\title{
Control of strand registry by attachment of PEG chains to amyloid peptides influences nanostructure
}

Article

Accepted Version

Castelletto, V., Cheng, G., Furzeland, S., Atkins, D. and Hamley, I. (2012) Control of strand registry by attachment of PEG chains to amyloid peptides influences nanostructure. Soft Matter, 8. pp. 5434-5438. ISSN 1744-683X doi: https://doi.org/10.1039/c2sm25546d Available at https://centaur.reading.ac.uk/27910/

It is advisable to refer to the publisher's version if you intend to cite from the work. See Guidance on citing.

To link to this article DOI: http://dx.doi.org/10.1039/c2sm25546d

Publisher: Royal Society of Chemistry

All outputs in CentAUR are protected by Intellectual Property Rights law, including copyright law. Copyright and IPR is retained by the creators or other copyright holders. Terms and conditions for use of this material are defined in the End User Agreement.

www.reading.ac.uk/centaur 
Central Archive at the University of Reading

Reading's research outputs online 


\section{Control of Strand Registry by Attachment of PEG Chains to Amyloid Peptides Influences Nanostructure}

Valeria Castelletto, ${ }^{1}$ Ge Cheng, ${ }^{1,3}$ Steve Furzeland, ${ }^{2}$ Derek Atkins, ${ }^{2}$ and Ian W. Hamley ${ }^{1, *}$

${ }^{1}$ Dept of Chemistry, University of Reading, Whiteknights, Reading RG6 6AD, UK

${ }^{2}$ Colworth Discover-Unilever R\&D, Sharnbrook MK44 1LQ, UK

${ }^{3}$ Currently at Department of Chemistry, University of Liverpool, Crown Street Liverpool L69 7ZD, UK

*Author for correspondence. I.W.Hamley@ reading.ac.uk

Communication, for Soft Matter

Revised 2/4/12 


\begin{abstract}
The self-assembly in aqueous solution of PEG-peptide conjugates comprising a model amyloid peptide sequence FFKLVFF that contains the A $\beta(16-20)$ KLVFF motif is investigated. X-ray diffraction reveals different packing motifs dependent on PEG chain length. This is correlated to remarkable differences in self-assembled nanostructures, depending on PEG chain length. The control of strand registry points to a subtle interplay between aromatic stacking, electrostatic and amphiphilic interactions.
\end{abstract}


The registry of strands within a $\beta$-sheet can have a profound influence on the structure of amyloid fibrils, including polymorphism which may be responsible for the prevalence of different prion strains. For example, strand registry within amyloid fibrils formed by natural proteins leads to distinct steric zipper structures, as revealed by X-ray microcrystallography. ${ }^{1}$ Strand registry has been studied in shorter amyloid peptides, including fragments of the amyloid $\beta(\mathrm{A} \beta)$ peptide using isotope-edited FTIR spectroscopy ${ }^{2,3}$ and ${ }^{13} \mathrm{C}$ solid state NMR methods. ${ }^{3,4}$ Recently, magic angle spinning NMR techniques have been introduced that can be used to measure intermolecular interactions via correlation spectra involving ${ }^{12 / 13} \mathrm{C}$ and/or ${ }^{14 / 15}$ N. ${ }^{5}$ Fiber X-ray diffraction has recently been used to deduce the registry of strands formed by variants of 12-residue peptide containing $\mathrm{K}$ and/or R, E, A, F residues. ${ }^{6}$

Attachment of polyethylene glycol (PEG) chains (PEGylation) to peptides leads to conjugates with enhanced stability with potential in biomedical applications as well as the possibility to create distinct self-assembled nanostructures. For example, PEG has been linked to $A \beta(10-35)$ fragments ${ }^{7,8}$ and the C-terminal attachment of PEG leads to inhibition of fibrillisation compared to the peptide alone. ${ }^{7}$ We have recently investigated the self-assembly of model PEG-peptide hybrids containing $A \beta(16-20)$, i.e. KLVFF-based sequences such as FFKLVFF, ${ }^{9-11} \beta A \beta A K L V F F{ }^{12}$ or YYKLVFF $^{13}$ with an N-terminal modification with two hydrophobic and aromatic residues and PEG attached at the $\mathrm{C}$ terminus. By appropriate choice of PEG chain length, ${ }^{11}$ favorable amphiphilicity is conferred and the formation of lyotropic liquid crystal phases at high concentration in aqueous solution is observed. ${ }^{9-11,13}$ 
Here, we show that strand registry in PEG-FFKLVFF conjugates is determined by the balance of interactions that control self-assembly in these hybrids, specifically aromatic $\pi$-stacking interactions involving phenylalanine residues, electrostatic interactions involving the $\mathrm{K}$ residue and the peptide terminus and amphiphilic interactions, i.e. the hydrophobic effect and the solubilization conferred by PEG. The FFKLVFF sequence is chosen due to its high hydrophobicity, because it contains four phenylalanine residues. Self-assembly of the peptide in methanol has been reported previously (it does not dissolve in water). ${ }^{14}$ Here, we report that changing PEG molar mass leads to a change in strand registry as revealed by fibre X-ray diffraction.

Three $(\mathrm{EG})_{\mathrm{n}}$-FFKLVFF-COOH conjugates were studied where EG denotes ethylene glycol and $n=5,11$ or 27 is the PEG degree of polymerization. These samples will be denoted $\mathrm{n} 5, \mathrm{n} 11$ and $\mathrm{n} 27$ in the following. The PEG chains were attached at the $\mathrm{N}$ terminus to further elucidate the effect of charge, in comparison with our previous work on C-terminal PEG congugates. ${ }^{9-13}$ To examine the effect of $\mathrm{N}$-terminal aromatic hydrophobic units a further three conjugates Fmoc-(EG) $)_{n}$-FFKLVFF-COOH were prepared. The Fmoc [Fmoc=N-(fluorenyl-9-methoxycarbonyl)] unit has been widely used in peptide systems to drive aggregation via $\pi$-stacking interactions. ${ }^{15}$ All conjugates were synthesized in house using methods described in the SI.

It was found that all three PEG-FFKLVFF conjugates were water soluble, however the three Fmoc-PEG-FFKLVFF conjugates were not fully soluble in water but were soluble in aqueous mixtures containing the polar solvent acetonitrile (as used in HPLC analysis) and therefore self-assembly of the latter conjugates was studied in water/acetonitrile mixtures. It is interesting that the addition of the single Fmoc unit 
can be used to tune solubility of these conjugates, and as shown below this moiety also has a significant effect on the self-assembly behaviour.

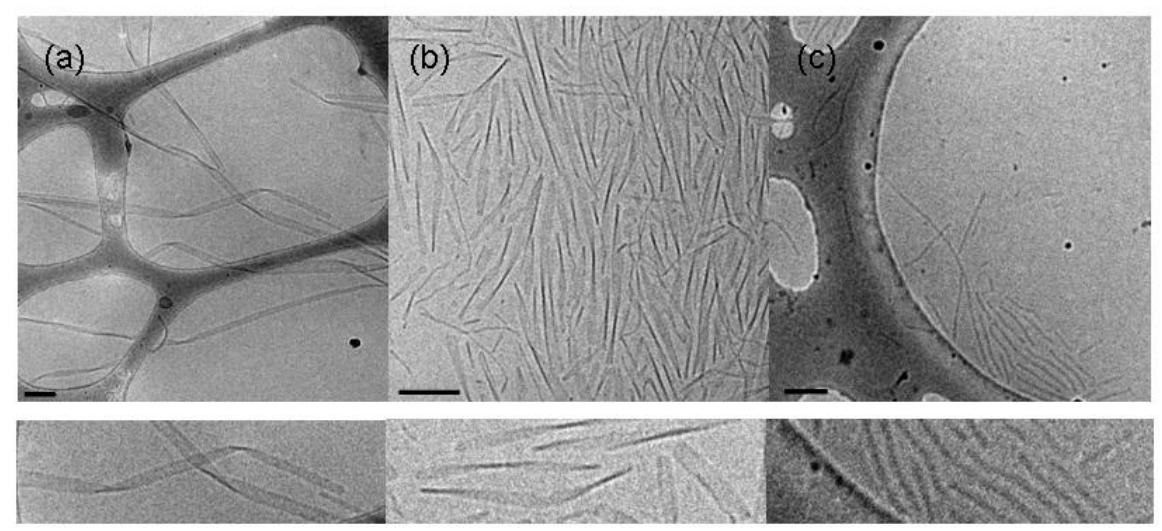

Figure 1. Cryo-TEM images. (a) $0.5 \mathrm{wt} \% \mathrm{n} 5$ in water, (b) $0.5 \mathrm{wt} \% \mathrm{n} 11$ in water, (c) 0.5 wt\% $\mathrm{n} 27$ in water. The scale bars indicate $200 \mathrm{~nm}$. The images below show enlargements of the self-assembled nanostructures.

Cryo-TEM images for the PEG-FFKLVFF conjugates in aqueous solution are shown in Fig.1 (additional images at a higher concentration are shown in SI Fig.1). These are very insightful and reveal that the morphology of self-assembled PEGFFKLVFF nanostructures depends on PEG chain length. For n5, extended (length extends to $\mu \mathrm{m}$ in some cases) twisted ribbons are observed. Conjugate $\mathbf{n} \mathbf{1 1}$, also forms twisted ribbons (Fig.1b), with a shorter length than those for $\mathbf{n 5}$. The conjugate with the longest PEG chain length, $\mathbf{n 2 7}$ on the other hand, forms uniform fibrils (Fig.1c) with a diameter of $(20 \pm 5) \mathrm{nm}$. In contrast to the images for the PEG-FFKLVFF samples, the Fmoc-PEG-FFKLVFF samples showed much less well defined (or no) self-assembled nanostructures in mixed solvent (1:1 or 2:1 acetonitrile:water) solutions. No structures were observed in a $1 \mathrm{wt} \%$ solution of n11-Fmoc and few 
well-defined structures were observed for the other two conjugates, although a few short fibril clusters were observed for CG-101Fmoc.

SAXS provided additional in situ data on the self-assembled structure of the PEGFFKLVFF samples. SI Fig. 2 shows SAXS profiles. The broad peak centred at $q=2.7$ $\mathrm{nm}^{-1}(d=2.3 \mathrm{~nm})$ is assigned to a spacing associated with the length of the $\beta$-strands This data also enables a clear distinction to be drawn between $\mathbf{n} 27$ and the other two samples, firstly because the overall shape of the scattering profile is different for this sample, reflecting the distinct morphology. As shown in SI Fig.2, the low q slope for $\mathbf{n} 27$ is $q^{-1}$ consistent with a cylinder form factor, however for $\mathrm{n} 5$ and $\mathrm{n} 11$ the slope is $q^{-2}$ consistent with sheets. Model form factors were used to obtain a fibril radius for $\mathbf{n} 27$ of $2.2 \mathrm{~nm}$ and a sheet thickness (modeled using a disk form factor) of $3 \mathrm{~nm}$ for $\mathbf{n 5}$ and n11. These values provide precise input into our model for the self-assembled structures to be discussed shortly. Note that for $\mathbf{n} 27$ the fibril diameter from cryoTEM is larger than the value obtained from the SAXS analysis which may be due to the exclusion of the hydrated PEG shell from the modeling of the SAXS data. In addition, $\mathbf{n} 27$ does not show a peak in the $q=2.5-3 \mathrm{~nm}^{-1}$ range in contrast to the other two. This feature is associated with the length of the extended peptide strand, as also observed by fibre X-ray diffraction and discussed in more detail in the following.
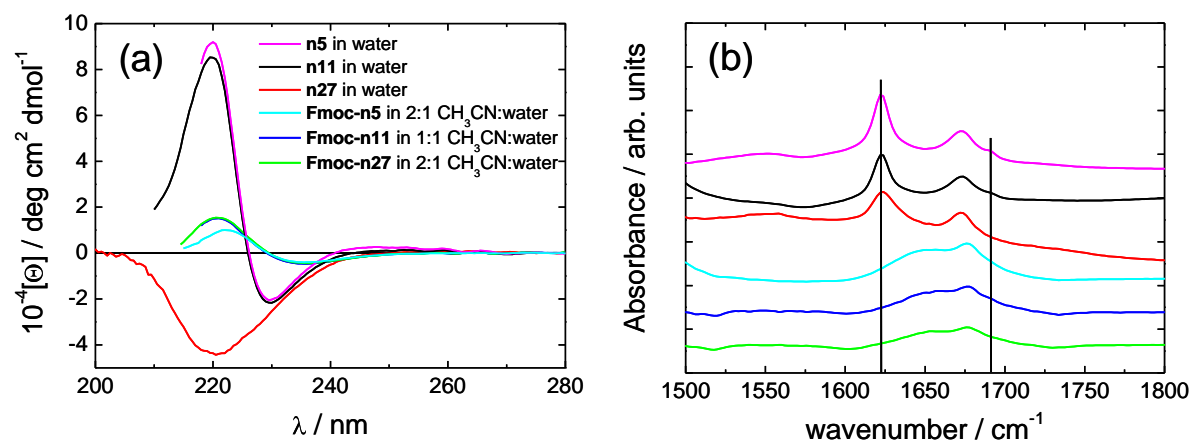
Figure 2. Spectra for conjugates (1\% solution in the indicated solvents). (a) CD spectra, (b) FTIR spectra in the amide I' region (same colour scheme to label samples as in part a). The vertical lines indicate $1622 \mathrm{~cm}^{-1}$ and $1691 \mathrm{~cm}^{-1}$.

The secondary structure was probed using CD and FTIR spectroscopies. The CD spectra in Fig.2a fall into two classes for the PEG-FFKLVFF and Fmoc-PEGFFKLVFF conjugates. In the former case, the spectra show $\beta$-sheet features, i.e. a minimum with negative ellipticity in the range $220-230 \mathrm{~nm}$ and a positive maximum at lower wavelength. Both these features are red-shifted compared to the usual peak minimum location, $216 \mathrm{~nm}$, due to aggregation. ${ }^{16}$ This is particularly evident for $\mathbf{n 5}$ and $\mathbf{n 1 1}$, red-shifting due to twisting of $\beta$-sheets being a known effect. ${ }^{16}$ The spectrum for $\mathbf{n} 27$ which forms fibrils and not twisted ribbons, most resembles a classical $\beta$ sheet spectrum. In contrast, the spectra for the Fmoc-PEG-FFKLVFF conjugates show positive maxima at $220 \mathrm{~nm}$. This is ascribed to aromatic stacking interactions. We have previously observed spectra with this feature for peptides ${ }^{17,18}$ and PEGpeptide conjugates $^{9}, 11,13,19$ containing phenylalanine residues and assigned it to aromatic stacking interactions of these residues. Here, the $\beta$-sheet ordering predominates for the PEG-FFKLVFF conjugates, but for the Fmoc-PEG-FFKLVFF conjugates, cryo-TEM indicates no or weak self-assembly (at the same $1 \mathrm{wt} \%$ concentration) and so aromatic stacking interactions dominate the electronic CD spectra. We suggest that the $220 \mathrm{~nm}$ positive maximum then arises from $\pi-\pi$ stacking interactions of phenylalanine and Fmoc residues.

FTIR spectra in the amide I' region (Fig.2b) support the interpretations from CD and cryo-TEM in that $\beta$-sheet features are observed for the three PEG-FFKLVFF 
conjugates, but not the Fmoc-PEG-FFKLVFF conjugates. The spectra for the three PEG-FFKLVFF show peaks at 1622 and $1691 \mathrm{~cm}^{-1}$ and these are assigned to $\beta$-sheet structure. The peak at $1672 \mathrm{~cm}^{-1}$ is assigned to bound TFA counterions. ${ }^{18}$ The presence of the (weak) $1691 \mathrm{~cm}^{-1}$ peak suggests that some or all $\beta$-sheets may have an antiparallel configuration. ${ }^{20}$ This is in agreement with our previously published spectra for FFKLVFF-PEG conjugates ${ }^{11}$ (although not our initial interpretation of data for FFKLVFF-PEG3k ${ }^{9}$ ) and with recent analysis of X-ray diffraction patterns from FFKLVFF-PEG2k. In contrast, the spectra for the Fmoc-PEG-FFKLVFF conjugates show no evidence of $\beta$-sheet features and just exhibit a maximum around $1650 \mathrm{~cm}^{-1}$ due to disordered/random coil structure. ${ }^{20}$
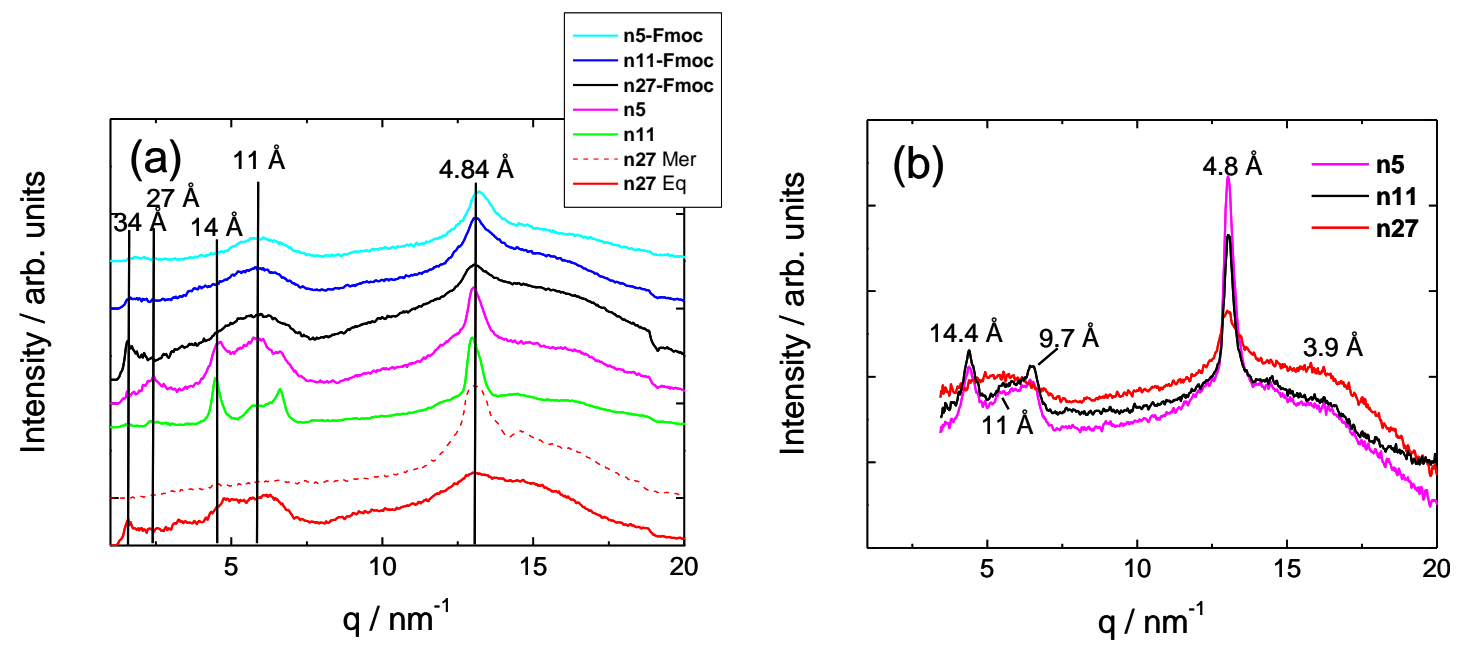

Figure 3. XRD intensity profiles (a) from sector averages obtained from fibre XRD patterns for dried stalks, samples as indicated. Due to the anisotropy observed in the pattern for $\mathrm{n} 27$ both equatorial and meridional sector intensity profiles are shown. (b) from synchrotron WAXS on $1 \%$ solutions.

Fibre X-ray diffraction was performed on aligned stalks dried from solution. Intensity profiles are shown in Fig.3a, these being obtained by sector integration of 
the 2D profiles in SI Fig.3. Corresponding $d$ spacings are listed in SI Table 1. These profiles reveal features associated with $\beta$-sheet ordering of the peptides for all samples, i.e. reflections are observed corresponding to the sheet spacing (range of $10.6-11.4 \AA$ ) and that of the $\beta$-strands within the sheets (range $4.83-4.86 \AA$ ). The diffraction patterns show only weak alignment of the $4.8 \AA$ reflection preferentially along the meridian but nevertheless these patterns are consistent with typical amyloid “cross- $\beta$ " XRD pattern features. ${ }^{21}$ As shown in Fig. 3a, additional reflections are observed for all three conjugates that lack the Fmoc unit, as listed in Table 1. These reflections are not observed for any of the three Fmoc homologues which do not exhibit an ordered secondary structure. The additional reflections are thus associated with the $\beta$-sheet ordering of the PEG-FFKLVFF conjugates. These long spacings have not previously been observed by us for FFKLVFF-PEG conjugates (with PEG molar mass in the range $\left.1-10 \mathrm{~kg} \mathrm{~mol}^{-1}\right){ }^{11}$ with the exception of a $d=17.6 \AA$ shoulder peak observed for FFKLVFF-PEG3k. ${ }^{22}$ It is notable that the peaks observed for the longer $\mathbf{n} 27$ conjugate here are distinct from those for the other two conjugates (which have a similar list of $d$ spacings). In addition, the XRD patterns for $\mathbf{n} 27$ exhibit a much higher degree of alignment than for the other two samples. We ascribe this to the absence of twisting of the fibrils (vide infra). These results indicate that additional peaks are revealed when the PEG molar mass is sufficiently low, and in the absence of the aromatic Fmoc unit. This points to an important role of the Fmoc unit in suppressing the ordering of $\beta$-sheets of the FFKLVFF peptide.

Remarkably, it proved possible to perform synchrotron WAXS on the PEGFFKLVFF conjugates in aqueous solution ( $1 \mathrm{wt} \%$ solutions). The corresponding intensity profiles are shown in Fig. 3b. This data provides essential support to the 
main findings from the fibre XRD experiments on dried stalks. In particular, the in situ solution WAXS profiles all contain a main 4.8 A peak associated with the spacing between peptide backbones within $\beta$-sheets. In addition, for $\mathbf{n 5}$ and $\mathbf{n 1 1}$, several additional reflections are observed at $14.4 \AA, 11 \AA$ and $9.7 \AA$. In contrast, for $\mathbf{n} 27$ a single broad peak corresponding to $d=12 \AA$ is observed. These spacings are in excellent agreement with the values obtained from the fibre diffraction patterns, and provide confirmation that the drying process during stalk preparation does not disrupt the secondary structure.

(a)

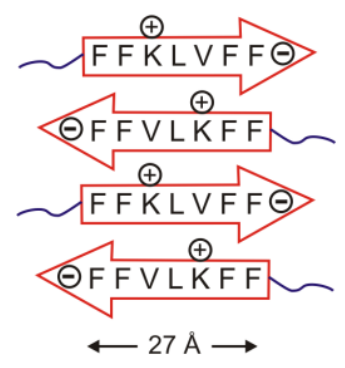

(b)

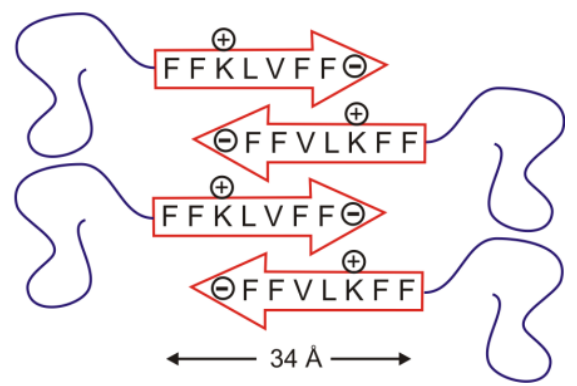

Figure 4. Proposed model for strand registry in (a) $\mathbf{n} 5$ and $\mathbf{n} 11,(\mathrm{~b}) \mathrm{n} 27$.

Taken together, the fibre XRD and in situ solution WAXS experiments confirm that $\mathbf{n 2 7}$ has a different packing of $\beta$-sheets than $\mathbf{n 5}$ or $\mathbf{n 1 1}$. This is related to the formation of distinct self-assembled structures, i.e. twisted ribbons for $\mathbf{n 5}$ and $\mathbf{n 1 1}$ and fibrils for $\mathbf{n 2 7}$. The $d=27 \AA$ spacing (with possible second order reflection at around $14 \AA$ ) for $\mathbf{n 5}$ and $\mathbf{n 1 1}$ is slightly longer than the length of a heptapeptide in an antiparallel configuration $^{23}(7 \times 3.4=24 \AA)$. We have adopted a model with $\beta$-sheets in an antiparallel configuration due to the observation of features in the FTIR spectra 
associated with antiparallel sheets. This leads to a model distinct from that previously proposed for FFKLVFF-PEG3k which comprised strands arranged radially within the core of the core-shell fibrils. ${ }^{9}$ The proposed model is shown in Fig. 4a.

In contrast, n27 shows a $34 \AA$ long spacing along with the characteristic 10-12 $\AA$ spacing associated with the stacking of $\beta$-sheets. Our model for this system consists of antiparallel $\beta$-strands offset by two residues, as shown in Fig. $4 \mathrm{~b}$ this is favoured by interactions between the positively charged lysine residue and the $\mathrm{N}$ terminus.

\section{(a) n5 and n11}

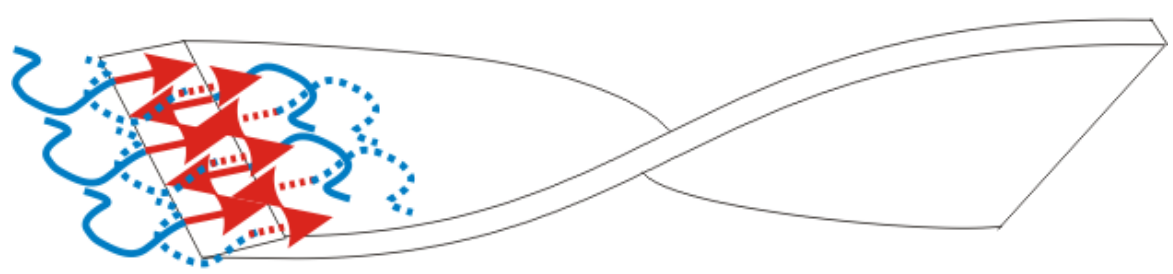

(b) $\mathrm{n} 27$

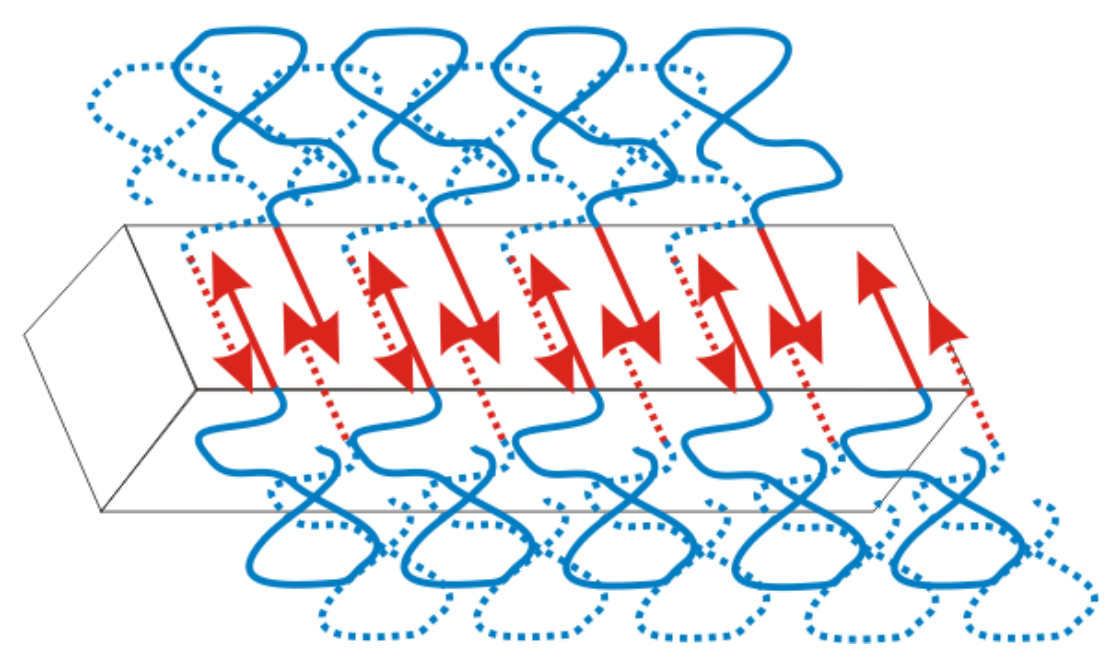

Figure 5. Proposed model for self-assembled nanostructures. For convenience, only two stacked $\beta$-sheets are shown in each case. 
Consideration of the nanostructures observed by cryo-TEM along with the strand registry probed by XRD leads to models for the self-assembled nanostructures shown in Fig.5. Cryo-TEM clearly shows that $\mathbf{n 5}$ and $\mathbf{n 1 1}$ form twisted sheets with considerable (polydisperse) width, but small thickness. We suggest that the thin sheets actually correspond to monolayers as sketched in Fig.5a, in order to keep the short PEG chains in contact with water. The twisting of the sheets leads to rotation of strands around the fibril axis and loss of orientation in the XRD patterns (SI Fig.3). For n27, the fibrils observed by cryo-TEM have a very small diameter and we associate this with the width of the PEG-peptide bilayer in Fig.4b (estimated to be approximately $10 \mathrm{~nm}$, assuming PEG chains are stretched to several times $R_{\mathrm{g}}{ }^{9}$ ). The stacking of the sheets is shown in a head-to-tail configuration. A high degree of strand alignment within the fibrils is expected, consistent with the observed XRD pattern. The shorter length of the $\mathbf{n} \mathbf{1 1}$ and $\mathbf{n} \mathbf{2 7}$ nanostructures might be associated with wrapping of the longer PEG chains around the tape/fibril "ends", i.e. with a reduced end-cap energy. An alternative, or possibly additional, explanation relies on the stretching energy penalty of the PEG chains, which can be released only by a reduction in fibril length as the molar mass of the PEG increases. ${ }^{24}$

These results show that the strand registry in PEG-peptides can be controlled by adjustment of PEG chain length. In addition, in PEG-peptide conjugates with lengthy PEG chains, the hydration of the hydrophilic PEG appears to be a larger influence on self-assembly than the hydrophobic/aromatic stacking interactions of $\mathrm{F}$ residues that governs the self-assembly for shorter PEG chains (Fig.4a). For $\mathbf{n 5}$ and $\mathbf{n 1 1}$, the tightly coupled strand registry shown in Fig.4a leads to tape-like objects. The persistence 
length is strongly dependent on PEG chain length, being much larger for the short $\mathbf{n 5}$ PEG chain where the peptide interactions predominate. In contrast, for $\mathbf{n} 27$ the longer hydrated PEG chain length gives greater conformational flexibility and self-assembly produces short fibrils.

In summary, both the self-assembled nanostructure and the strand-registry are influence by PEG chain length. The $\beta$-strand registry also depends on the presence of suitably charged residues in the peptide sequence. These findings highlight the use of PEGylation to control strand registry of $\beta$-sheet forming peptides, and the design criteria (appropriate positioning of hydrophobic and charged residues) to achieve a desired strand offset. In addition, our results point to the important influence of terminal aromatic units such Fmoc on the self-assembly of PEG-peptide conjugates. 


\section{References}

1 M. R. Sawaya, S. Sambashivan, R. Nelson, M. I. Ivanova, S. A. Sievers, M. I. Apostol, M. J. Thompson, M. Balbirnie, J. J. W. Wiltzius, H. T. McFarlane, A. O. Madsen, C. Riekel, and D. Eisenberg, Nature, 2007, 447, 453.

S. A. Petty and S. M. Decatur, Proc. Natl. Acad. Sci. U. S. A., 2005, 102, 14272; S. M. Decatur, Acc. Chem. Res., 2006, 39, 169; Y. Liang, S. V. Pingali, A. S. Jogalekar, J. P. Snyder, P. Thiyagarajan, and D. G. Lynn, Biochemistry, 2008, 47, 10018.

A. K. Mehta, K. Lu, W. S. Childers, S. Liang, J. Dong, J. P. Snyder, S. V.

Pingali, P. Thiyagarajan, and D. G. Lynn, J. Am. Chem. Soc., 2008, 130, 9829.

T. L. S. Benzinger, D. M. Gregory, T. S. Burkoth, H. Miller-Auer, D. G.

Lynn, R. E. Botto, and S. C. Meredith, Biochemistry, 2000, 39, 3491; W. S.

Childers, A. K. Mehta, R. Ni, J. V. Taylor, and D. G. Lynn, Angew. Chem. Int. Ed. Engl., 2010, 49, 4104.

M. J. Bayro, G. T. Debelouchina, M. T. Eddy, N. R. Birkett, C. E. MacPhee, M. Rosay, W. E. Maas, C. M. Dobson, and R. G. Griffin, J. Am. Chem. Soc., 2011, 133, 13967; J. R. Lewandowski, P. C. A. van der Wel, M. Rigney, N. Grigorieff, and R. G. Griffin, J. Am. Chem. Soc., 2011, 133, 14686. K. E. Marshall, K. L. Morris, D. Charlton, N. O'Reilly, L. Lewis, H. Walden, and L. C. Serpell, Biochemistry, 2011, 50, 2061.

Meredith, and D. G. Lynn, J. Am. Chem. Soc., 1998, 120, 7655. P. Thiyagarajan, J. Am. Chem. Soc., 1999, 121, 7429. 
I. W. Hamley, M. J. Krysmann, V. Castelletto, A. Kelarakis, L. Noirez, R. A. Hule, and D. Pochan, Chem. Eur. J., 2008, 14, 11369; I. W. Hamley , M. J. Krysmann, V. Castelletto, and L. Noirez, Adv. Mater., 2008, 20, 4394.

I. W. Hamley, M. J. Krysmann, G. E. Newby, V. Castelletto, and L. Noirez, Phys. Rev. E, 2008, 57, 062901.

V. Castelletto, G. E. Newby, Z. Zhu, I. W. Hamley, and L. Noirez, Langmuir, 2010, 26, 9986.

V. Castelletto, J. M. E. McKendrick, I. W. Hamley, C. Cenker, and U. Olsson, Langmuir, 2010, 26, 11624.

V. Castelletto, G. E. Newby, D. Hermida-Merino, I. W. Hamley, D. Liu, and L. Noirez, Polymer Chemistry, 2010, 1, 453. M. J. Krysmann, V. Castelletto, and I. W. Hamley Soft Matter, 2007, 3, 1401. Z. Yang and B. Xu, Chem. Comm., 2004, 2424; V. Jayawarna, M. Ali, T. A. Jowitt, A. F. Miller, A. Saiani, J. E. Gough, and R. V. Ulijn, Adv. Mater., 2006, 18, 611; A. Mahler, M. Reches, M. Rechter, S. Cohen, and E. Gazit, Adv. Mater., 2006, 18, 1365; G. Cheng, V. Castelletto, R. Jones, C. J. Connon, and I. W. Hamley, Soft Matter, 2011, 7, 1326; D. M. Ryan, S. B. Anderson, and B. L. Nilsson, Soft Matter, 2010, 6, 3220; D. J. Adams, Macromol. Biosci., 2011, 11, 160. I. W. Hamley, D. R. Nutt, G. D. Brown, J. F. Miravet, B. Escuder, and F. Rodríguez-Llansola, J. Phys. Chem. B, 2010, 114, 940. M. J. Krysmann, V. Castelletto, A. Kelarakis, I. W. Hamley, R. A. Hule, and D. J. Pochan, Biochemistry, 2008, 47, 4597; V. Castelletto, I. W. Hamley , P. J. F. Harris, U. Olsson, and N. Spencer, J. Phys. Chem. B, 2009, 113, 9978; V. Castelletto, I. W. Hamley, R. A. Hule, and D. J. Pochan, Angew. Chem., Int. 
Ed. Engl., 2009, 48, 2317; V. Castelletto, I. W. Hamley , C. Cenker, and U. Olsson, J. Phys. Chem. B, 2010, 114, 8002.

V. Castelletto, I. W. Hamley, C. Cenker, U. Olsson, J. Adamcik, R.

Mezzenga, J. F. Miravet, B. Escuder, and F. Rodriguez-Llansola, J. Phys.

Chem. B, 2011, 115, 2107.

V. Castelletto and I. W. Hamley Biophys. Chem., 2009, 141, 169.

A. Barth, Biochim. Biophys. Acta-Bioenerg., 2007, 1767, 1073.

E. D. Eanes and G. G. Glenner, J. Histochem. Cytochem., 1968, 16, 673; H. Inouye, P. E. Fraser, and D. A. Kirschner, Biophys. J., 1993, 64, 502; L. C. Serpell, Biochim. Biophys. Acta, 2000, 1502, 16; T. R. Jahn, O. S. Makin, K. L. Morris, K. E. Marshall, P. Tian, P. Sikorski, and L. C. Serpell, J. Mol. Biol., 2010, 395, 717.

I. W. Hamley and M. J. Krysmann, Langmuir, 2008, 24, 8210.

T. E. Creighton, 'Proteins. Structures and Molecular Properties', W.H.Freeman, 1993.

P. A. Rühs, J. Adamcik, S. Bolisetty, A. Sanchez-Ferrer, and R. Mezzenga, Soft Matter, 7, 3571. 